\title{
Optionen bei diastolischer Herzinsuffizienz
}

\section{Zwei Studien versprechen neue Therapieoptionen bei diastolischer Herzinsuffizienz: Ein Mineralkorti- koid-Rezeptor-Antagonist und ein Angiotensin Rezeptor Neprilysin In- hibitor.}

Bei mehr als der Hälfte aller Fälle von Herzinsuffizienz liegt die Ursache in einer diastolischen Compliance-Störung, häufig auf dem Boden einer Hypertonie, während die systolische Pumpfunktion noch weitgehend erhalten ist. Die Lebenserwartung dieser Patienten ist reduziert, evidenzbasierte Therapien sind rar. Aldosteron kann in der Pathogenese eine Rolle spielen, denn das Steroidhormon fördert Fibrose und Hypertrophie des Myokards sowie die Versteifung der Gefäße.

Die Arbeitsgruppe um den Kardiologen Prof. Burkert Pieske aus Graz untersuchte in der doppelblinden Aldo-DHFStudie (Aldosterone Rezeptor Blockade in Diastolic Heart Failure) den Nutzen einer Spironolacton-Therapie. Die $422 \mathrm{~Pa}$ tienten befanden sich in einem noch frühen Stadium der diastolischen Herzschwäche. Primäre Endpunkte waren die diastolische Herzfunktion sowie die Belastbarkeit nach 12 Monaten.

\section{Herzfunktion gebessert, Belastbarkeit unverändert}

Es zeigte sich, dass Spironolacton (25 mg/d) den Blutdruck signifikant senkte. Außerdem wurden eine Reihe von Parametern der diastolischen Herzfunktion und Herzstruktur signifikant gebessert, unabhängig von der Blutdrucksenkung, betonte Pieske. Auch das NT-proBNP sank als Ausdruck reduzierter neuroendokriner Aktivität. Auf die Belastbarkeit, das Herzinsuffizienz-Studium oder auf die Lebensqualität hingegen hatte die Behandlung keinen Einfluss. Spironolacton wurde generell gut vertragen. Allerdings erlitten einige Patienten eine Gynäkomastie, eine Anämie oder eine Verschlechterung der Nierenfunktion.

Ein weiterer therapeutischer Ansatzpunkt könnte die bei diastolischer Relaxa- tionsstörung verminderte natriuretische Antwort auf akute Volumenbelastung sein. Vor diesem Hintergrund mag ein Medikament sinnvoll sein, welches die Aktivität des endogenen natriuretischen Peptid-Systems steigert. Ein solches ist LCZ696, das von Novartis klinisch entwickelt wird. Das Medikament hat einen dualen Wirkmechanismus: Zum einen hemmt es den Abbau von natriuretischem Peptid und steigert dessen Aktivität. Zum anderen enthält es Valsartan und blockt somit den Angiotensin-1-Rezeptor.

\section{Wie sieht's nach zwölf Wochen aus?} Die Wirksamkeit von LCZ696 (bis zu $2 \times 200 \mathrm{mg} / \mathrm{d}$ ) wurde in der PARAMOUNT-Studie bei 308 Patienten mit Herzinsuffizienz und erhaltener Ejektionsfraktion im Vergleich zu Valsartan (bis zu 2x160 mg/d) untersucht. Alle Patienten waren symptomatisch und wiesen deutlich erhöhte NT-proBNP-Werte (> 400 pg/ml) auf. Primärer Endpunkt war die Wirkung auf NT-proBNP nach 12-wöchiger Behandlung, sekundäre Endpunkte waren echokardiografische Parameter der diastolischen Herzfunktion.

Wie Prof. Dr.Scott Solomon, Brigham and Women's Hospital der Harvard Medical School in Boston, berichtete, reduzierte LCZ696 die NT-proBNP nach 12 Wochen um 23\% deutlicher als Valsartan $(\mathrm{p}=0,005)$. Auch nach 36 Wochen hatte die NT-proBNP-Reduktion noch Bestand, wenngleich nach dieser Zeit der Unterschied zu Valsartan (-15\%) nicht mehr signifikant war. Gleichzeitig wurde das Volumen des linken Vorhofes reduziert. Beide Parameter sind eindeutig mit der Prognose dieser Krankheit assoziiert, sagte Solomon. Auch die NYHA-Klasse verbesserte sich unter LCZ696.

Die Verträglichkeit war sehr gut. Monotherapien mit ACE-Hemmern oder AT1-Blockern hatten in größeren Studien bei diastolischer Herzinsuffizienz bisher nur einen Trend zu klinischer Verbesserung ergeben.

(Dirk Einecke) $\frac{\text { Hier steht eine Anzeige }}{\text { This is an advertisement }}$ 